\title{
Flexible Width Nyquist Pulse Based on a Single Mach-Zehnder Modulator
}

\author{
Jianqi Hu, Simon J. Fabbri", Camille-Sophie Brès \\ Ecole Polytechnique Fédérale de Lausanne, Photonic Systems Laboratory (PHOSL), STI-IEL, Station 11, CH-1015 Lausanne, Switzerland \\ *simon.fabbri@epfl.ch
}

\begin{abstract}
We present a Nyquist pulse generation technique based on a single Mach-Zehnder modulator driven by a multi-harmonic electrical signal. The direct control of the RF components yields a range of $10 \mathrm{GHz}$ sinc-shaped pulse train.

OCIS codes: (230.2090) Electro-optical devices; (320.5540) Pulse shaping ; (230.6080) Sources
\end{abstract}

\section{Introduction}

To keep pace with the growing demands for network traffic, many transmission methods are investigated to optimize the capacity of the existing optical fibre communication links. Super-channels orthogonally multiplexed allows for the optimum utilization of the bandwidth offered by installed optical fibres [1,2]. The generation of Nyquist pulse in the optical domain is explored for transmission system in order to supplant the limited bandwidth and large order finite impulse response (FIR) filter required electronically [3].

One of the pulse shape matching the Nyquist criterion is of particular interest due to its minimum spectral occupancy: the sinc-shaped pulse, often defined as a raised-cosine with a roll-off equal to zero. Spectrally the Nyquist pulse corresponds to a rectangular shaped optical frequency combs with equal amplitude and linear spectral phase. Varieties of techniques have been proposed for the generation of Nyquist pulse train. The simplest and most cost-effective technique relies on external modulation of $\mathrm{CW}$ laser, and does not require line-by-line shaping nor band pass filtering. Sinc-shaped Nyquist pulses had been reported from setup consisting of multiple Mach-Zehnder modulators (MZM) in series [4] or dual-parallel Mach-Zehnder modulator (DP-MZM) driven by high RF power [5].

In this paper, we propose an alternative simple method for generating sinc-shaped Nyquist pulses based on a single MZM. Synchronized multi-harmonic RF signals are combined to modulate an optical carrier. By using from 2 to 4 RF signals, we report 4- to 9-line comb Nyquist pulses.

\section{Principle and experiment results}

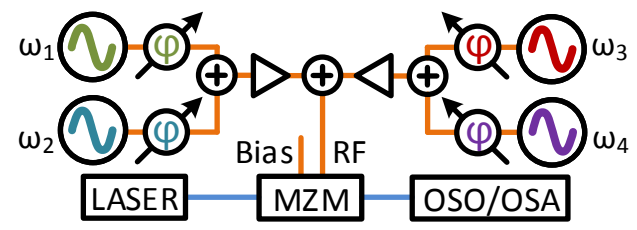

Fig. 1: Schematic of the multi-harmonic Nyquist source. OSO: Optical Sampling Oscilloscope, OSA: Optical Spectrum Analyzer, $\phi$ : tuneable delay line.

$$
\begin{array}{r}
E_{\text {out }}(t)=E_{0} \cos \left(\varphi+\beta_{k} \sum_{k=1}^{N} \cos \omega_{k} t\right) e^{i \omega_{0} t} \\
E_{\text {out }}(t) \approx-\frac{\beta}{2} E_{0} \sum_{k=1}^{N}\left(e^{i(k-1 / 2) \omega_{m} t}-e^{-i(k-1 / 2) \omega_{m} t}\right) e^{i \omega_{0} t}
\end{array}
$$

The conceptual setup (Fig. 1) consists in a single MZM driven by synchronized RF clock sources [6]. The use of multi-harmonic comb source provides the control of each pair of sidebands and hence the perfect flatness of the optical comb alongside the high side-mode suppression ratio (SMSR) characteristics of the Nyquist spectrum. The electric field at the output of a single MZM driven by $N$ synchronized RF signals is given by Eq. (1), where $\varphi=\pi V_{\text {bias }} / V_{\pi 0}$ is the normalized DC bias voltage and $\beta_{k}=\pi V_{\mathrm{k}} / 2 V_{\pi \mathrm{k}}(k=1,2, \ldots N)$ are the modulation indices.

By keeping $\beta_{k}$ of each RF components equal and small, the output of the MZM driven by $\omega_{m}, 2 \omega_{m}, \ldots N \omega_{m}$ multi-harmonics can be approximated by Eq. (2). In order to obtain equal amplitude and equal phase of all optical frequency components $\cos \varphi=-\beta \sin \varphi / 2$ has to be satisfied. Hence, sinc-shaped Nyquist pulses, with $(2 N+1)$ spectral lines spaced by $\omega_{m}$ is generated. Similarly, to achieve even number tones Nyquist pulses, the frequencies $\omega_{m} / 2,3 \omega_{m} / 2, \ldots(2 N-1) \omega_{m} / 2$ are used to drive the modulator biased at its minimum transmission point $(\varphi=\pi / 2)$ for carrier suppression, giving Eq. (3). It can be seen that $2 N$ comb lines matching Nyquist condition is obtained, also at the repetition rate of $\omega_{m}$.

The experimental setup of the reconfigurable optical Nyquist pulse source was composed of an external cavity laser tuned at $1549.8 \mathrm{~nm}$ with an output power of $13 \mathrm{dBm}$, followed by a commercial $30 \mathrm{GHz}$ low V $\pi \mathrm{MZM}$ driven 
by multiple synchronized RF signals. The modulator output signal was split equally to simultaneously measure the spectrum and the temporal waveform. Because of the low sensitivity of the $500 \mathrm{GHz}$ optical sampling oscilloscope (OSO), the optical signal was amplified to $4.1 \mathrm{dBm}$. A $5 \mathrm{~nm}$ filter was added before the OSO to limit the amplified spontaneous emission noise received.

The electrical part of the setup consists of 4 independent RF clock sources of various bandwidth, RF broadband amplifiers, RF power combiners, and electrical delay lines as phase control. Similarly, a single clock source can be used followed by a bank of RF frequency multipliers. For 5, 7, 9-line comb generation, 2 to 4 RF frequencies were used with $10 \mathrm{GHz}$ fundamental frequency and its harmonics, i.e. $20 \mathrm{GHz}, 30 \mathrm{GHz}$, and $40 \mathrm{GHz}$. While for 4, 6, 8 spectral lines, 2 to $4 \mathrm{RF}$ frequencies were set to $5 \mathrm{GHz}, 15 \mathrm{GHz}, 25 \mathrm{GHz}$, and $35 \mathrm{GHz}$. The relative phase of these RF components was fully tunable either directly from the clock source or by inserting electrical delay lines on the paths. The repetition rate is maintained at $10 \mathrm{GHz}$ in all cases reported here as consequence of the frequency doubling obtained by biasing the modulator at null.

Fig. 2 presents a selection of the pulse trains and corresponding optical spectra, obtained by modulating the MZM by an electrical signal composed of multiple RF components equally spaced and in phase. Time waveforms show a very close shape compared to the ideal Nyquist pulse. The spectra also show characteristic rectangular shape with a flatness of $0.09,0.12$, and $0.15 \mathrm{~dB}$ and a SMSR of 30.4, 11.2, and $23.9 \mathrm{~dB}$ for the 7, 8, and 9-line comb respectively. The quality of the optical comb obtained is dependent of the total RF power because a high driving signal generates higher orders side-bands and hence will distort the comb shape [7]. On the contrary, a low RF power signal results in lower pulse power at the optical output. Fig. $2 \mathrm{~b}$ illustrates the trade-off between the pulse shape generated and the total output power by comparing the full width at half maximum at different RF power.
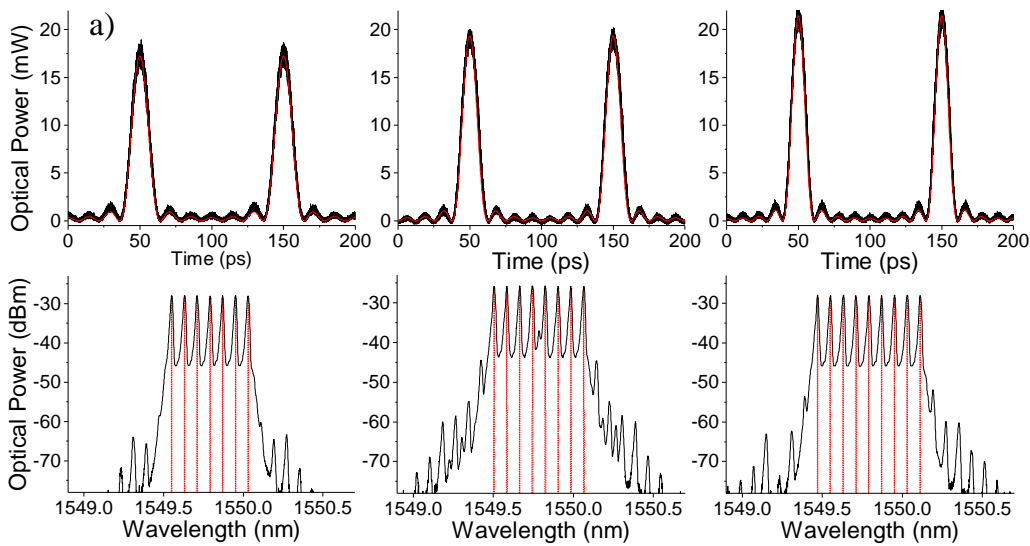

b)

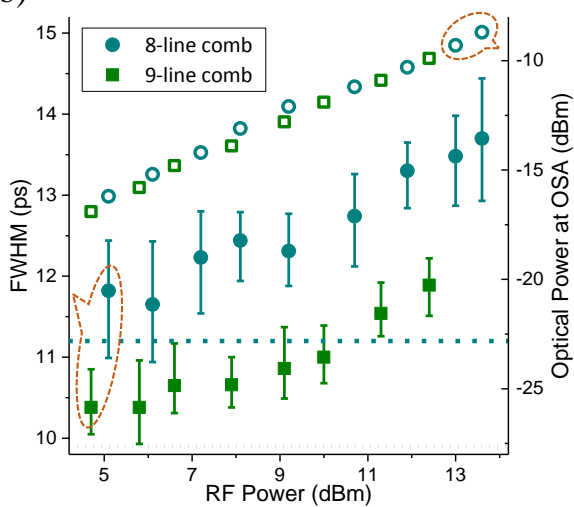

Fig. 2: a) Selection of Nyquist pulses and corresponding optical spectra for 3 and 4 RF frequencies. Left and Right are using a 10GHz and harmonics signal, center is using $5,15,25$, and $35 \mathrm{GHz}$ components signal. The power of the modulator driving signal was 6.3, 10.8, and 8.6 $\mathrm{dBm}$, respectively. Red: Simulated ideal sinc-shaped Nyquist pulses and corresponding spectra.

b) Full Width at Half Maximum (Full; min and max values) and optical power measured at the OSA (Hollow) vs. the total RF power. Square: 9-line comb, Circle: 8-line comb. Dotted line: ideal 9-line FWHM, dashed line: ideal 8-line FWHM.

We have demonstrated a simple method for the generation of flexible sinc-shaped pulses. By using multiharmonic synchronized RF signals to drive a single MZM, the full control is given over the repetition rate, the duty cycle, and the central wavelength without optical filter or wave-shaper. By using from 2 to 4 RF clock sources, we report 4- to 9-line comb Nyquist pulses at a constant repetition rate of $10 \mathrm{GHz}$, with the associated clear rectangular optical comb spectrum.

This work was supported by the Swiss National Science Foundation under Grant 159897.

\section{References}

[1] D. Klonidis et al., "Enabling Transparent Technologies for the Development of Highly Granular Flexible Optical Cross-Connects," Proc. ICTON, We.D1.5, Graz (2014).

[2] M. Nakazawa et al., "Ultrahigh-Speed "Orthogonal” TDM Transmission with an Optical Nyquist Pulse Train,” Opt. Express, Vol. 20 , p. 1129 (2012).

[3] J. Wang et al., "Generation of Spectrally Efficient Nyquist-WDM QPSK Signals Using Digital FIR or FDE Filters at Transmitters," J.

Lightwave Technol., Vol. 30, no. 23, p. 3679-3686 (2012).

[4] M. A. Soto et al., "Optical Sinc-Shaped Nyquist Pulses of Exceptional Quality," Nat. Comm., vol. 4, p. 2898 (2013).

[5] Q. Wang et al., "Ultra-Flat Optical Frequency Comb Generator Using a Single-Driven Dual-Parallel Mach-Zehnder Modulator," Opt. Lett., Vol. 39, p. 3050 (2014).

[6] S. J. Fabbri et al., "Multi-Harmonic Optical Comb Generation," Proc. ECOC, Mo.2.A.2, Amsterdam (2012).

[7] M. McCarthy et al., "Signal Processing Using Opto-Electronic Devices," in All-Optical Signal Processing, S. Wabnitz S and B. J. Eggleton, ed. (Springer Series in Optical Sciences, vol. 194. Springer, 2015). 